\title{
Arcabouço Conceitual de Adaptação de Recursos Educacionais para Recomendação de Arquiteturas Pedagógicas
}

\author{
Vitor Bremgartner ${ }^{1,2}$, José Francisco M. Netto ${ }^{1}$, Crediné Menezes ${ }^{3}$ \\ ${ }^{1}$ Instituto de Computação (IComp) - Universidade Federal do Amazonas (UFAM) \\ Av. Gen. Rodrigo Otávio, Coroado. Manaus - AM - Brasil \\ ${ }^{2}$ Instituto Federal do Amazonas (IFAM) - Campus Manaus Distrito Industrial \\ Av. Danilo Areosa, Distrito Industrial. Manaus - AM - Brasil \\ ${ }^{3}$ Faculdade de Educação - Universidade Federal do Rio Grande do Sul (UFRGS) \\ Av. Paulo Gama, Farroupilha. Porto Alegre - RS - Brasil \\ \{vitorbref, jnetto\}@icomp.ufam.edu.br, credine@gmail.com
}

\begin{abstract}
Typically, the existing resources in Virtual Learning Environments (VLEs), used in distance education courses and blended, are presented in the same way for all students. This may complicate the effective learning process of each student. The approach adopted for the solution of this problem in this paper is based on a framework called ArCARE, which allows adaptation of resources for students in VLEs and student monitoring during his course, allowing the construction of his knowledge, using multiagent system technology that handles an open learner model ontology. These ArCARE resources may be recommendation and adaptation of Pedagogical Architectures for the students have a more effective learning of a particular course content. Results obtained in a discipline of Algorithms and Programming show the feasibility of the proposal.
\end{abstract}

Palavras-Chave: Arquiteturas Pedagógicas, Ambientes Virtuais de Aprendizagem, Adaptação de Recursos.

Resumo. Normalmente, os recursos existentes em Ambientes Virtuais de Aprendizagem (AVAs), utilizados em cursos de educação a distância e em semipresenciais, são apresentados da mesma forma para todos os alunos. Isto pode dificultar o processo de aprendizagem efetiva de cada aluno. A abordagem adotada para a solução deste problema neste artigo baseia-se em um arcabouço chamado ArCARE, que permite adaptação de recursos para alunos em AVAs e o acompanhamento do aluno no decorrer do seu curso, permitindo a sua construção do conhecimento, utilizando a tecnologia de sistema multiagente que manipula uma ontologia de modelo aberto de aluno. Um dos recursos do ArCARE é a recomendação e adaptação de Arquiteturas Pedagógicas para os alunos terem uma aprendizagem mais efetiva do conteúdo de um determinado curso. Resultados obtidos em uma disciplina de Algoritmos e Programação mostram a viabilidade da proposta.

Keywords: Pedagogical Architectures, Virtual Learning Environments, Resources Adaptation.

\section{Introdução}

A Educação a Distância (EaD) já é uma modalidade amplamente utilizada nos processos de ensinoaprendizagem. Para apoiar os cursos de EaD ou semipresenciais existem os Ambientes Virtuais de Aprendizagem (AVAs), facilitando o processo de comunicação entre estudantes, professores, tutores e a comunidade, permitindo a todos participarem de um modo interativo com a disponibilidade de materiais didáticos [Bremgartner, Netto e Menezes 2015]. Além disso, existem AVAs que utilizam uma camada de inteligência, especialmente no que diz respeito à possibilidade de ensino flexível para os alunos, em que o ambiente de aprendizagem é capaz de adaptar os seus recursos 
V Congresso Brasileiro de Informática na Educação (CBIE 2016)

Anais dos Workshops do V Congresso Brasileiro de Informática na Educação (CBIE 2016)

apresentados de modo a alcançar uma aprendizagem mais efetiva, de acordo com as necessidades do aluno. Ferramentas na área da Inteligência Artificial, tais como ontologias e agentes de software podem atuar integrados nestes AVAs, sendo responsáveis por esta camada de inteligência e fazendo uso de um modelo do aluno [Viccari e Giraffa 2003].

A adaptação de recursos em AVAs pode ser entendida como a adaptação do que é apresentado para o aluno no AVA, como atividades passadas, objetos de aprendizagem, mudanças na interface e auxílio personalizado de acordo com o modelo de aluno, obtido através de seu perfil. Este modelo é um registro das ações dos alunos, bem como a informação útil a respeito do aluno no ambiente de aprendizagem. Por exemplo, estes dados podem ser informações pessoais, preferências e desempenho em atividades. Instituições e organizações internacionais têm estabelecido a padronização de modelo de aluno utilizado em AVAs, sendo que o padrão mais conhecido atualmente é o Instructional Management Systems Learning Information Package (IMS LIP) [LIP 2016].

No entanto, apesar da crescente utilização de ambientes educacionais, eles geralmente oferecem recursos de aprendizagem da mesma forma para todos os alunos, resultando que a aprendizagem pode não se tornar efetiva para todos, devido às diversas características cognitivas que cada aluno possui. Isto, portanto, cria dificuldades de aprendizagem para alguns alunos ou até mesmo a falta de interesse por parte dos alunos no uso do ambiente de aprendizagem.

Sendo assim, a abordagem adotada para solução deste problema neste artigo baseia-se em um arcabouço chamado ArCARE (Arcabouço Conceitual de Adaptação de Recursos Educacionais), sendo uma estratégia que permite adaptação de recursos para alunos em AVAs e o acompanhamento do aluno no decorrer do seu curso, com base no Construtivismo de Piaget, utilizando a tecnologia de sistema multiagente que manipula uma ontologia de modelo aberto de aluno composto de várias características que o aluno possui, tais como competências, habilidades, equipamentos que o aluno utiliza, o desempenho nas atividades, frequência e estilos de aprendizagem. Os recursos de adaptação no ArCARE são a recomendação de Arquiteturas Pedagógicas (APs), atividades, vídeos e leituras, com o objetivo de o aluno ter uma aprendizagem mais efetiva do conteúdo de um determinado curso.

Nessa proposta, quanto ao modelo de aluno, este é alterado dinamicamente durante o curso, através das interações do aluno com o AVA. O modelo de aluno também é apresentado ao estudante, sendo um Modelo Aberto de Aluno (MAA). Usar MAA refere-se a tornar o modelo de aluno explícito ao estudante, de modo a fornecer um recurso adicional através do autoconhecimento e possível autorregulação do processo de aprendizagem, com o objetivo de melhorar a aprendizagem e autonomia do aluno [Bull, Quigley e Mabbott 2006]. Quanto às Arquiteturas Pedagógicas, sua compreensão na área educacional tem trazido múltiplas interpretações. Iremos seguir a concepção elaborada por [Carvalho, Nevado e Menezes 2005], em que APs podem ser compreendidas como "estruturas de aprendizagem realizadas a partir da confluência de diferentes componentes: abordagem pedagógica, software, Internet, inteligência artificial, educação a distância, concepção de tempo e espaço".

Além desta Introdução, o artigo está estruturado da seguinte forma: a Seção 2 mostra alguns conceitos e trabalhos relacionados. A Seção 3 apresenta uma visão geral do modelo conceitual do ArCARE, descrevendo também o sistema multiagente e o modelo de aluno. A Seção 4 apresenta testes realizados de adaptação de recursos com base em APs na disciplina de Algoritmos e Programação em um AVA. A Seção 5 apresenta as conclusões e trabalhos futuros.

\section{Adaptação de Recursos e Arquiteturas Pedagógicas em AVAs}

O uso de práticas construtivistas relacionadas com o modelo de aluno em AVAs tem sido uma alternativa utilizada para apoiar a tomada de decisões nos processos de ensino e aprendizagem mediados pela tecnologia, como a adaptação de recursos de aprendizagem.

$\mathrm{Na}$ literatura pesquisada, identificamos trabalhos com adaptação de recursos utilizando Arquiteturas Pedagógicas (APs). Em [Marcon, Machado e Carvalho 2012], é explorada a capacidade 
V Congresso Brasileiro de Informática na Educação (CBIE 2016)

Anais dos Workshops do V Congresso Brasileiro de Informática na Educação (CBIE 2016)

de utilizar o Facebook para a elaboração de APs com atividades em grupo. Por sua vez, em [Panceri e Menezes 2014] é utilizada a AP do debate de teses e são identificadas mediações pedagógicas que podem ser praticadas em AVAs por meio de processamento de linguagem natural, recuperação de informação e um sistema multiagente denominado ALPES.

Assim, podemos ver que existem estudos na literatura que lidam com adaptação de recursos, características construtivistas e utilização de APs em AVAs. A proposta do ArCARE contribui ao considerar várias características dos estudantes ao mesmo tempo, variando de acordo com a interação dos alunos com o AVA e também ao apresentar o modelo de aluno aberto para o mesmo, a fim de obter um diagnóstico mais preciso da situação dos alunos no ambiente educacional e estimulando-os a aprender cada vez mais, além de proporcionar a recomendação de recursos. A próxima seção descreve o modelo do ArCARE com mais detalhes.

\section{Modelo Conceitual do ArCARE}

O modelo conceitual do ArCARE é mostrado na Figura 1. Neste modelo, é mostrado em detalhes o arcabouço que constitui no processo de personalização do AVA por meio da recomendação de recursos educacionais nele, em função das características do aluno, fazendo uso de agentes de sofware e uma ontologia que descreve o modelo aberto de aluno.

Cada elemento composto neste modelo conceitual da Figura 1, que possui uma numeração para cada item, faz parte do processo de recomendação de recursos educacionais. Nesse arcabouço, consideramos que os usuários (estudantes, professores, administradores) estão sempre em interação com o AVA (1). O estudante acessa recursos, atualiza seus dados cadastrais, executa atividades propostas pelo professor, acessa o seu MAA, a fim de saber seu desempenho em meio ao curso a fim de fazer autorreflexões. O professor pode elaborar atividades, lançar notas, inserir recursos no repositório de recursos do AVA (6), além de executar outras ações conforme suas atribuições. Já os administradores são os responsáveis pela configuração do ambiente, conforme suas atribuições que lhe são designadas no próprio AVA.

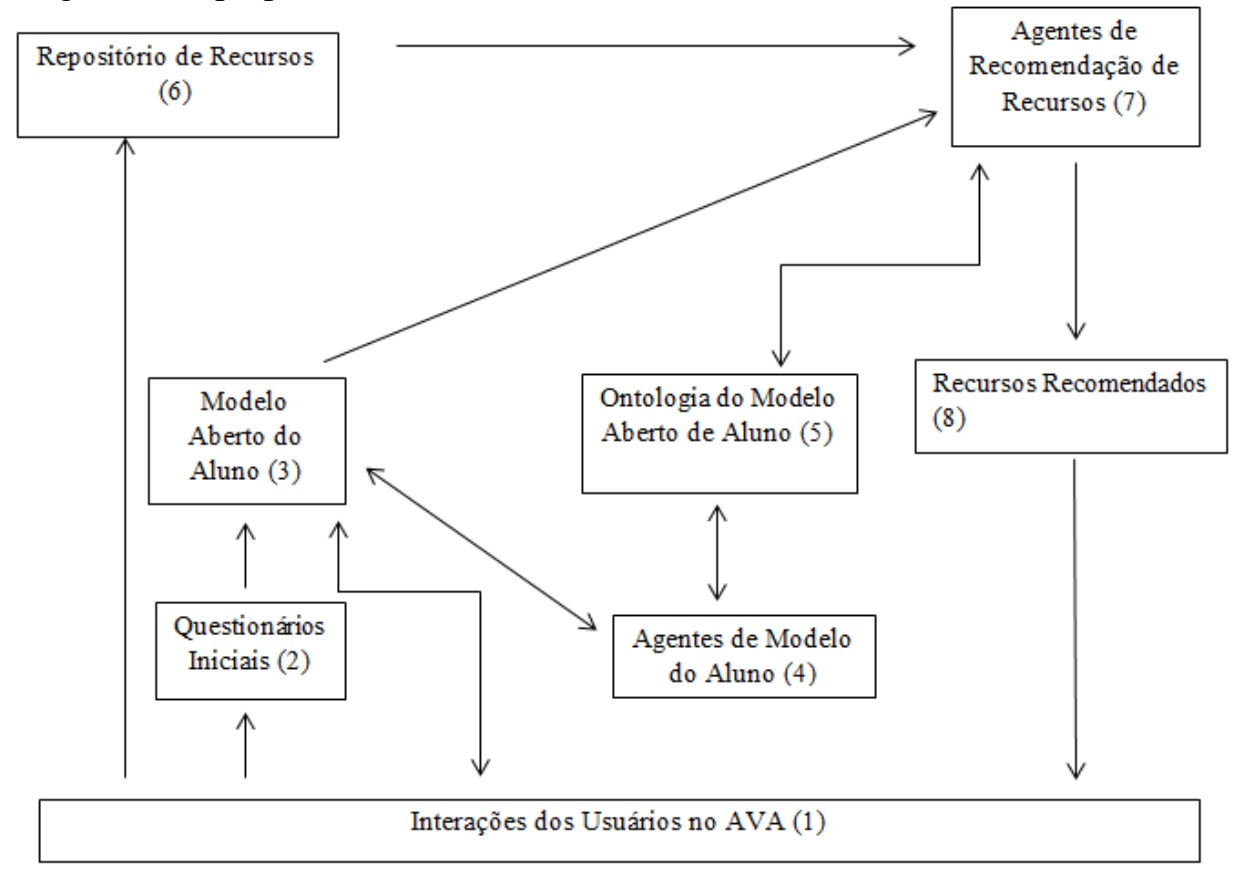

Figura 1. Modelo Conceitual do ArCARE.

Neste modelo conceitual mostrado na Figura 1, definimos que o aluno precisa ter o seu perfil inicial, ou seja, os dados que compõem seu modelo inicial, a fim de que o ambiente possa começar a ser adaptado, ao invés deste ter que esperar por diversas manifestações do estudante. Para isso, são passados questionários iniciais ao aluno (2), tais como perguntas para identificação de 
estilos de aprendizagem, testes iniciais para diagnóstico de competências do estudante e questões pessoais de acordo com o curso que ele estiver cursando.

De posse dessas informações, é inicializado o modelo do aluno (3), que é atualizado a cada interação do estudante com o AVA, por meio dos agentes que fazem a verificação em cima desse modelo, chamados agentes de modelo do aluno (4). Ao manipularem os dados dos alunos, estes agentes utilizam uma ontologia que descreve o seu modelo, a ontologia de modelo aberto do aluno (5), que contém regras para trocas de mensagens entre agentes, além de definições contidas no modelo do aluno.

Por sua vez, os agentes de recomendação de recursos (7) selecionam os recursos (6) que consideram mais adequados para cada estudante no curso ou disciplina, utilizando os dados do aluno (3) e a ontologia do modelo do aluno (5). Por fim, esses recursos são recomendados (8) para os alunos utilizarem no AVA (1). Percebe-se que ocorre um processo cíclico de recomendação e adaptação de recursos, uma vez que novos recursos são recomendados a partir que o modelo de aluno (3) é atualizado.

\subsection{O Sistema Multiagente}

A Figura 2 mostra a arquitetura interna de um agente do ArCARE, baseada no trabalho de [Netto, 2006]. A diferença de funcionalidades tanto entre os agentes de modelo de aluno quanto os de recomendação de recursos está no núcleo, pois neste estão identificados e implementados os propósitos que os agentes devem executar. A arquitetura interna visa satisfazer as funcionalidades levantadas para um agente no ArCARE. Sendo assim, na Figura 2 é apresentada a arquitetura interna do agente, que é baseada em três componentes básicos: o Núcleo do Agente, a Base de Conhecimento e o Elemento de Comunicação. Descrevemos a seguir os componentes propostos nesta arquitetura interna.

- Núcleo do Agente: realiza os processamentos, as inferências, acessa e atualiza a Base de Conhecimento e ativa o envio e o recebimento de mensagens no padrão Agent Communication Language - Foundation for Intelligent Physical Agents (ACL - FIPA) [FIPA 2016] para o Elemento de Comunicação. O Núcleo do Agente procura decodificar a semântica das mensagens recebidas, usando conhecimentos ontológicos registrados na Base de Conhecimento que comporta a Ontologia de Modelo Aberto do Aluno, e por meio das informações armazenadas na Base de Conhecimento que comporta os dados do banco de dados do AVA.

- Base de Conhecimento: subdivide-se em Dados do AVA e Ontologia de Modelo Aberto de Aluno. Os Dados do AVA constituem em um banco de dados onde ficam armazenadas todas as informações referentes ao AVA, que contém os dados dos perfis dos alunos, atividades, além de todas as interações dos usuários com o ambiente e todos os recursos disponíveis. Por sua vez, a Ontologia de MAA define os significados dos termos usados pelos agentes nas trocas de mensagens.

- Elemento de Comunicação: responsável por enviar e receber as mensagens trocadas entre os agentes. Ao enviar mensagens, utiliza o formato ACL FIPA.

Quanto ao núcleo dos agentes de recomendação, este contém elementos baseados na arquitetura BDI (Beliefs-Desires-Intentions) [Georgeff et al. 1999] para que seja executado o processo de recomendação de recursos.

\subsection{A Ontologia de Modelo Aberto de Aluno}

Outra importante etapa neste trabalho foi construir a ontologia de domínio que representa o modelo de aluno. Para o modelo de aluno foi utilizado o padrão IMS LIP [LIP 2016], que é um padrão largamente usado hoje em dia e que permite extensões. Originalmente, o LIP tem 11 categorias (ou classes) de dados do estudante, mas apenas cinco foram usadas (Activity, Competency, Identification, Accessibility e Interest). Além disso, este padrão foi estendido para as necessidades deste trabalho, em três outras categorias: CCPP_Device, Learning_Styles e Frequency. A categoria CCPP_Device 
V Congresso Brasileiro de Informática na Educação (CBIE 2016)

Anais dos Workshops do V Congresso Brasileiro de Informática na Educação (CBIE 2016)

descreve os dispositivos utilizados pelos alunos, no padrão Composite Capabilities/Preference Profile (CC/PP) [CC/PP 2016]. Usando a categoria Frequency, esta descreve a frequência dos alunos no AVA e também é verificado se o aluno terminou ou não suas atividades. Finalmente, a categoria Learning_Styles contém informações sobre os estilos de aprendizagem de FelderSilvernam e Honey-Alonso.

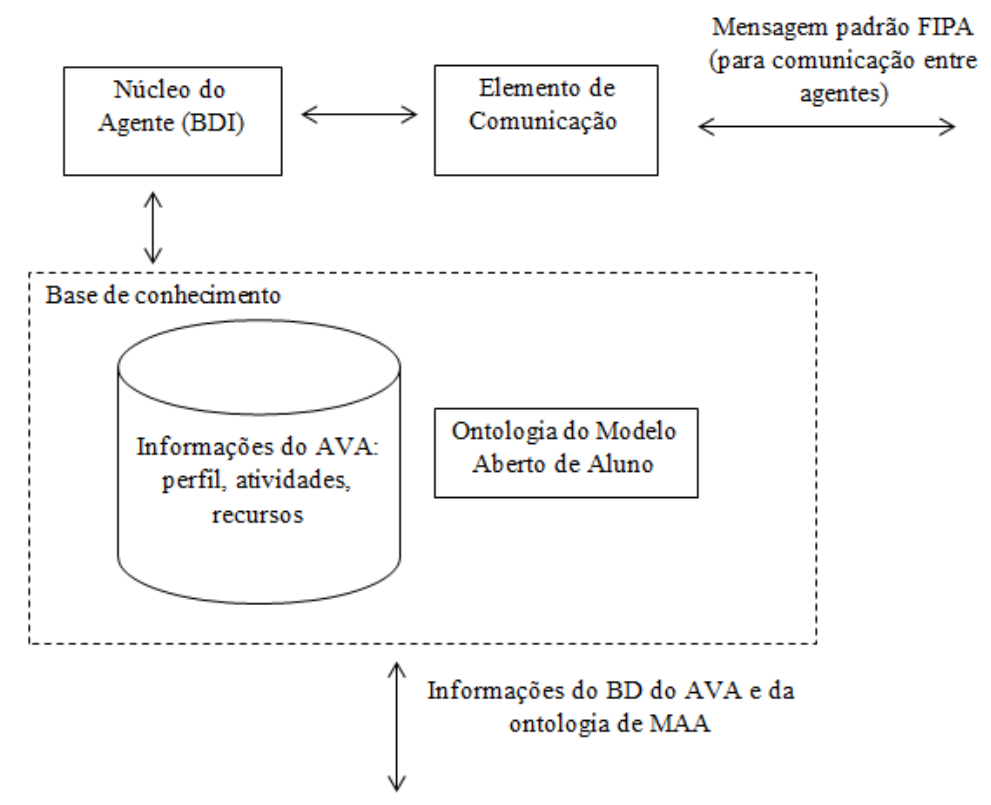

Figura 2. Arquitetura Interna de um Agente do ArCARE.

Na categoria Activity estão as informações acerca das atividades propostas pelo professor. As atividades, muitas neste trabalho baseadas em Arquiteturas Pedagógicas, possuem metadados para documentá-las utilizando o padrão LTSC/IEEE Learning Object Metadata (LOM) [IEEE 2002]. Esses metadados são úteis para o mapeamento feito entre os campos do LOM nas Arquiteturas Pedagógicas e os estilos de aprendizagem do aluno, a fim de que as APs recomendadas aos alunos sejam mais precisas. Neste caso, as APs podem ser vistas como objetos de aprendizagem. Sendo assim, os campos do LOM que fazem parte das características das Arquiteturas Pedagógicas estão de acordo com um subconjunto dos campos de metadados selecionado a partir do trabalho de [Araújo et al. 2014], o qual propôs um mapeamento dos campos de metadados com relação às caraterísticas dos estilos de aprendizagem do aluno. A ontologia de modelo de aluno na classe Competency descreve o nível de habilidade de cada aluno, o que é útil para identificar os alunos e seus níveis de competências e habilidades em uma disciplina ou curso. Nesse trabalho, cada competência do aluno é composta por um conjunto de habilidades específicas. Os agentes de modelo de aluno fazem avaliações frequentes das atividades dos alunos, sendo uma avaliação formativa, obtendo seu desempenho e atualizando os níveis de habilidades dos alunos. Outras categorias utilizadas são Identification (para os dados pessoais do aluno), Accessibility (dados de acessibilidade do usuário, credenciais no AVA) e Interest (contendo os interesses dos estudantes).

A ontologia de modelo de aluno foi desenvolvida no editor Protégé [Protégé 2016] e tem três classes principais: Concept, cujas filhas são as subclasses AID (descrevendo os agentes que fazem parte do sistema multiagente) e Learner_LIP (contendo as categorias LIP utilizadas neste trabalho, além de CCPP_Device, Learning_Styles e Frequency); Predicate, responsável pelos fatos sobre o ambiente onde os agentes atuam; e AgentAction (contendo as ações que os agentes realizam no AVA). Todas estas três classes permitem que a ontologia seja integrada ao sistema multiagente. A Figura 3 mostra uma representação gráfica resumida da ontologia de MAA, onde aparece apenas a subclasse Learner_LIP de Concept, que descreve o padrão LIP estendido e apresenta os slots (propriedades ou atributos) das demais classes. Por exemplo, na classe Learning_Styles, uma 
V Congresso Brasileiro de Informática na Educação (CBIE 2016)

Anais dos Workshops do V Congresso Brasileiro de Informática na Educação (CBIE 2016)

propriedade é $f s_{-} l s \_a c t i v e \_r e f l e c t i v e$, indicando se o aluno tende a ser mais ativo ou reflexivo nos estilos de aprendizagem de Felder-Silvernam.

Os 2 agentes (subclasses de AID) apresentados na Figura 3 são o Retrieved Profiles Agent e Recommended Resources Agent, que são agentes de recomendação de recursos. As relações e regras entre as classes descrevem os facets da ontologia, representados por setas (onde as setas com a relação "isa" significam "é um", de subclasse para superclasse). Como alguns exemplos de facets em setas azuis na Figura 3, o estudante faz uma atividade (doesActivity) e tem dúvidas ou erros (hasDoubts_Errors). Estes dois facets ligam as classes Identification e Activity. Além disso, o Recommended Resources Agent recomenda recursos (recommends_resources), por exemplo, APs de acordo com o perfil do estudante e as atividades que mais se ajustam a um determinado perfil de aluno (gets_best_fit_resources, que vem da classe Activity).

\section{Aplicação do ArCARE e Experimentos}

Nessa aplicação, utilizamos o AVA Moodle [Moodle 2016]. Nele, foram criados três questionários para os alunos responderem no início do curso, com o objetivo de obter o perfil e o modelo de aluno inicial, porque esta é a primeira informação que compõe a base de conhecimento sobre os alunos no banco de dados. Os questionários são: 1) Índice de Estilos de Aprendizagem (Index of Learning Styles), buscando conhecer os estilos de aprendizagem de Felder-Silvernam [Felder e Silverman 1988] dos estudantes; 2) Questionário de Estilos de Aprendizagem de HoneyAlonso [Alonso e Gallego 2016]. Optou-se por escolher estes dois questionários visando ter uma informação mais precisa a respeito dos alunos quanto aos seus estilos de aprendizagem, obtidos pela combinação dos dois questionários; 3) Questionário sobre algoritmos e programação de computadores, onde os alunos respondem a perguntas sobre o seu nível de conhecimento em conceitos de programação referentes a disciplina de Algoritmos e Programação (estruturas condicionais; repetição; estruturas de dados; nível de experiência no uso da linguagem de programação da disciplina - a linguagem $\mathrm{C}$; experiência de trabalhar com linguagens de programação no ramo profissional). Os links para acessar estes questionários e para acessar o MAA ficam disponíveis no AVA.

Por sua vez, os agentes de modelo de aluno foram desenvolvidos no framework baseado em Java JADE [JADE 2016], que manipulam os dados dos alunos e seus modelos de aluno no banco de dados do AVA. Já os agentes de recomendação de recursos, desenvolvidos no framework JADEX [JADEX 2016], que é uma extensão do JADE, a partir dos dados obtidos do modelo de aluno e usando a arquitetura BDI (Beliefs-Desires-Intentions), selecionam recursos contidos no repositório (que fica no banco de dados), como por exemplo, APs, para adaptar o AVA. Assim, o aluno acessa o AVA adaptado em funçao de seu perfil e o seu MAA. Os agentes de modelo do aluno são: Initial Profile Agent (que configura os níveis de competências, habilidades e estilos de aprendizagem iniciais dos alunos em função das respostas dos alunos dos 3 questionários respondidos no início do curso); Learning Assessment Agent (que avalia das atividades respondidas pelos alunos); Update Profile Agent (que atualiza os dados dos modelos de alunos mediante as interações dos mesmos com o ambiente, mediante as informações oriundas do Initial Profile Agent, Learning Assessment Agent $\mathrm{e}$ Frequency Evaluation Agent); Notifier Activity Agent (notificador de novas atividades passadas pelo professor, a fim de manter o aluno informado acerca de novas atividades); Frequency Evaluation Agent (que verifica a frequência do aluno no ambiente); Device Identifier Agent (que detecta informações a respeito do equipamento que o estudante está utilizando, a fim de ajustar o AVA de acordo com as características do dispositivo que o estudante estiver utilizando).

Os dois agentes de recomendação de recursos aos alunos no AVA são: Retrieved Profiles Agent (que faz a busca por dúvidas, erros dos alunos em atividades, avalia os níveis de habilidades e competências dos alunos, analisa seus estilos de aprendizagem, além das demais informações que compõem o MAA e por fim, mapeia esses dados em recursos de aprendizagem a serem disponibilizados no AVA, enviando uma mensagem ao Recommended Resources Agent); Recommended Resources Agent (que obtém as informações do Retrieved Profiles Agent e executa o processo de mostrar no AVA os recursos para cada aluno em função de seu MAA. Estes recursos 
V Congresso Brasileiro de Informática na Educação (CBIE 2016)

Anais dos Workshops do V Congresso Brasileiro de Informática na Educação (CBIE 2016)

podem ser: colegas que possam tirar dúvidas, notificações de acompanhamento do aluno, sugestão de leituras e atividades baseadas em arquiteturas pedagógicas).

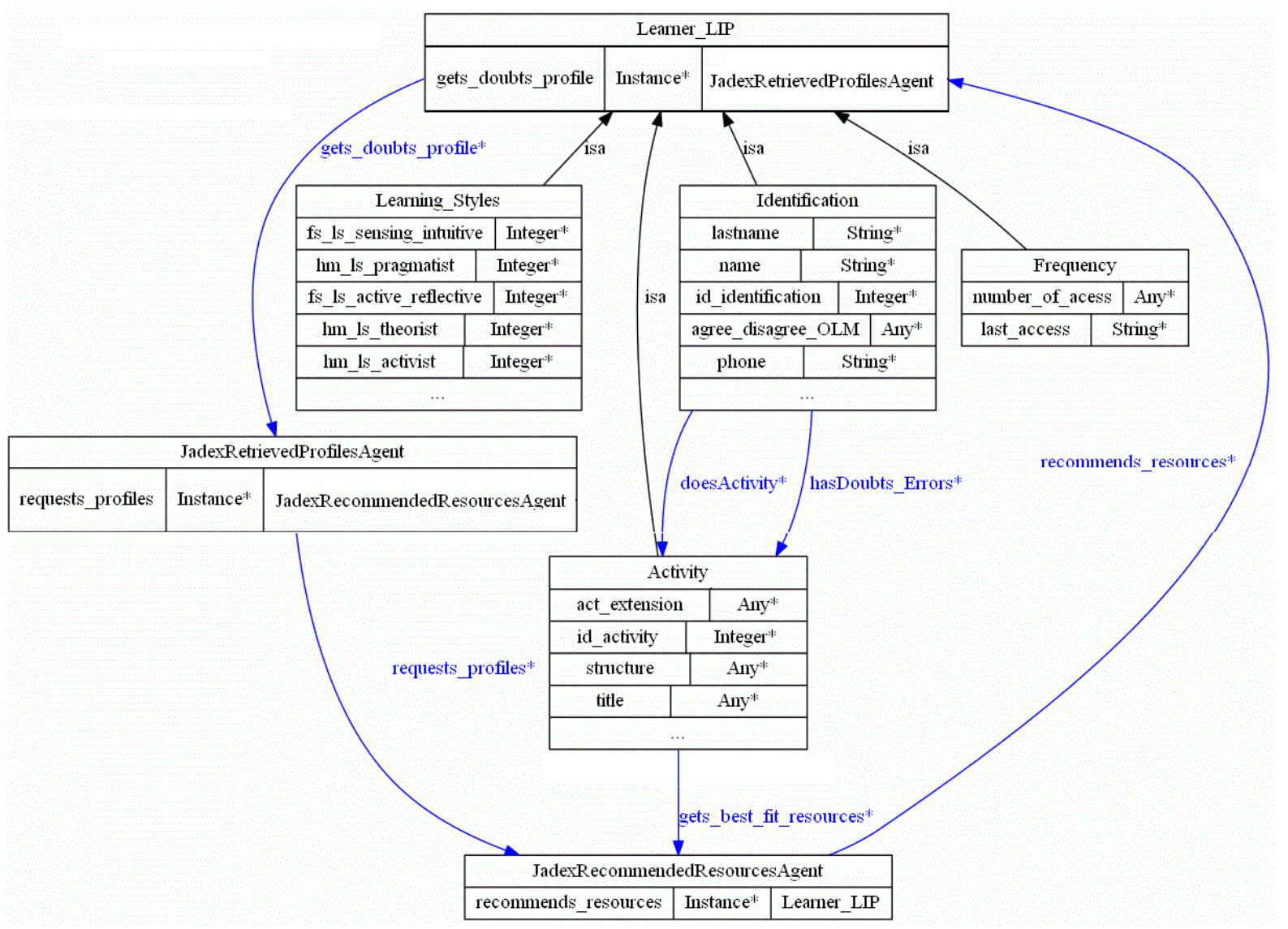

Figura 3. Representação gráfica resumida da Ontologia de Modelo Aberto de Aluno.

O aluno pode acessar seu modelo por meio de um link no AVA. É importante que o MAA seja facilmente compreendido pelo aluno. Assim, a Figura 4 mostra um exemplo de MAA apresentado aos alunos composto de três gráficos, que é o resultado das respostas dos alunos aos questionários. No primeiro gráfico são apresentados os estilos de aprendizagem de acordo com o questionário de Honey-Alonso, em porcentagens dos estilos Ativo, Reflexivo, Teórico e Pragmático. No segundo, os estilos de Felder-Silvernam (Ativo/Reflexivo, Sensorial/Intuitivo, Visual/Verbal e Sequencial/Global) são apresentados em valores ímpares que variam de $-11 \mathrm{a}+11$. Por exemplo, se um aluno tem no campo Visual/Verbal valor +7, isso significa que ele é mais Visual. Se fosse -7, ele seria mais Verbal. Finalmente, o terceiro gráfico mostra as habilidades do aluno em um curso específico, no nosso estudo de caso, Algoritmos e Programação.

No Moodle utilizado para testes, as adaptações de recursos (nesse caso as APs) foram em uma turma composta por 49 alunos da disciplina de Algoritmos e Programação no Instituto Federal do Amazonas Campus Manaus Distrito Industrial (IFAM-CMDI). Com a obtenção do perfil inicial de cada estudante a partir dos questionários de estilos de aprendizagem, foram recomendadas atividades de acordo com seus perfis. A Figura 5 mostra um exemplo de um debate de teses recomendado para alguns alunos, com a avaliação da resposta de um aluno por seu colega. No Moodle, a atividade do tipo Laboratório de Avaliação (originalmente chamada de Workshop) pode ser ajustada para funcionar como um debate de teses. Na atividade de Laboratório de Avaliação do Moodle, a adaptação ocorre primeiramente utilizando a heurística de que pares de alunos para um debate de teses podem ser formados com alunos de perfis heterogêneos, conforme indicado em [Castro e Menezes, 2011]. Inicialmente, os perfis foram diferenciados por estilos de aprendizagem, mas as outras características dos alunos descritas em seus modelos também podem ser usadas. É 
V Congresso Brasileiro de Informática na Educação (CBIE 2016)

Anais dos Workshops do V Congresso Brasileiro de Informática na Educação (CBIE 2016)

importante ressaltar que estas recomendações de atividades são feitas pelo Recommended Resources Agent. Pode-se perceber que a recomendação de recursos educacionais, neste caso, atividades baseadas em arquiteturas pedagógicas por meio de agentes e ontologia de MAA, é uma técnica construtivista útil para melhorar o aprendizado do aluno e que incentiva a prática de atividades colaborativas em AVAs.

Os alunos participantes dos testes foram questionados sobre as atividades realizadas. Alguns dos depoimentos foram: "com a correção dos meus colegas aprendi mais"; "eu ajudei e fui ajudado com muitas correções"; "A avaliação das atividades dos colegas foi feita de forma bem coerente a mim testando meus conhecimentos e verificando o conhecimento dos meus colegas"; "primeira vez que presencio uma didática dessa forma, seria excelente se as demais matérias fossem dessa forma"; "Gostei, pois foi feito de maneira individual e ao mesmo tempo pudemos compartilhar conhecimento, e perceber que podemos chegar ao mesmo resultado usando métodos diferentes."; "eu achei interessante, principalmente porque vim zerado de algoritmos e linguagem $C$ e tive a capacidade de avaliar alguém que já conhecia, pelo nível que foi a resposta dele e acho que ele conhecia mais do que eu"; "nós que somos avaliados, é bom (...) estar na situação tipo professor podendo avaliar os colegas e poder interagir com as diferentes respostas que iremos encontrar"; "achei interessante para ver a dificuladde de cada aluno e assim, (...) poder ajudar (...) formando um único conhecimento geral". Uma crítica feita pelos alunos foi quanto à interface dos debates de teses apresentados no AVA.

Meu Modelo Aberto de Aluno

Seu nome:

Gráfico dos seus estilos de aprendizagem de Honey-Mumford a partir do modelo de Kolb(Niveis em \%):

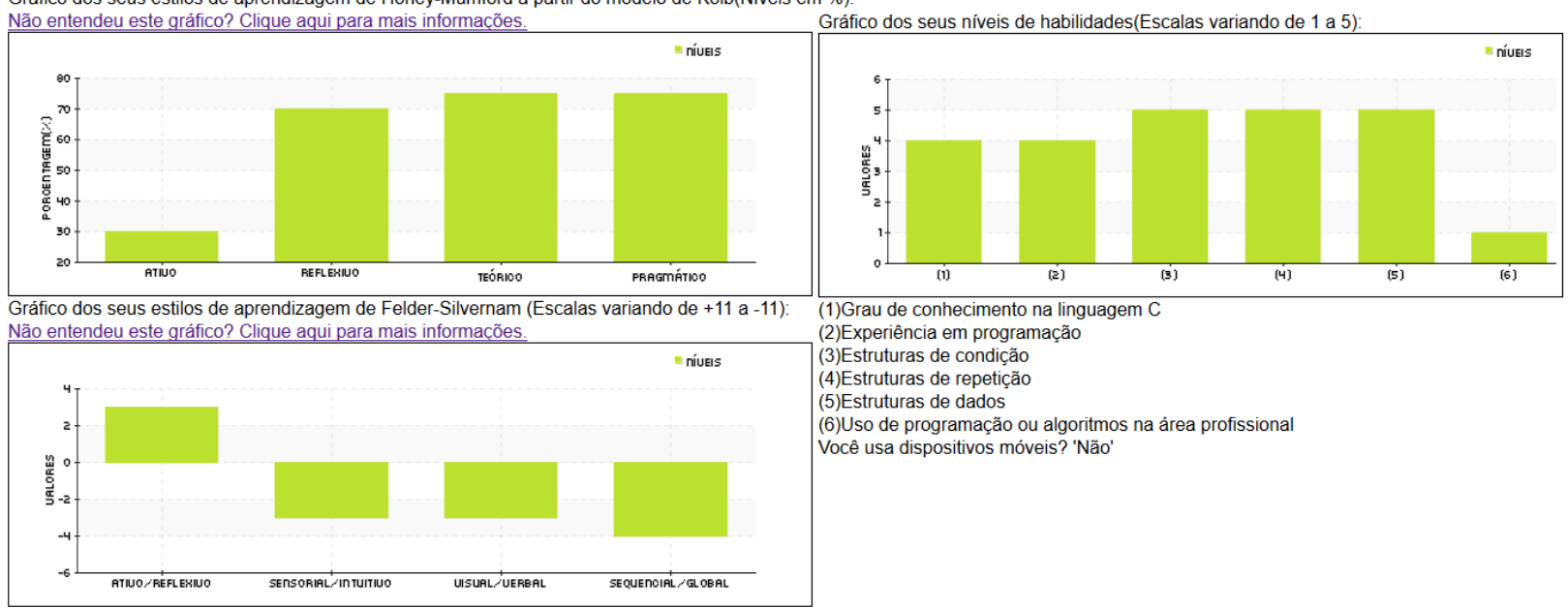

Figura 4. Apresentando o MAA aos estudantes.

\section{Conclusões e Trabalhos Futuros}

Este artigo mostrou o arcabouço ArCARE, que é um modelo de adaptação de recursos em AVAs baseado na teoria construtivista da aprendizagem. Foram desenvolvidos um sistema multiagente e uma ontologia de Modelo Aberto de Aluno no padrão IMS LIP capazes de selecionar recursos educacionais adaptados no AVA Moodle, como por exemplo, APs. Esta estratégia permite uma maior personalização dos recursos apresentados em função das características dos alunos. O processo de adaptação descrito na arquitetura do sistema aumenta a interação entre os alunos de um mesmo curso ou disciplina e a interação dos alunos com o AVA, aumentando assim o aprendizado efetivo e maiores acertos nas tarefas executadas.

Os testes realizados mostraram que a recomendação de APs é uma solução que pode ajudar a construir o conhecimento do aluno de forma útil e eficaz. A abordagem de sistema multiagente, juntamente com o MAA, pode ser aplicada a outros AVAs, uma vez que os agentes e o ambiente educacional podem compartilhar a mesma base de dados. Como trabalhos futuros, a ideia é testar o sistema para adaptação de conteúdo em dispositivos móveis, visto que 
V Congresso Brasileiro de Informática na Educação (CBIE 2016)

Anais dos Workshops do V Congresso Brasileiro de Informática na Educação (CBIE 2016)

a ontologia já descreve os recursos do dispositivo móvel no padrão CC/PP, fazer ajustes na interface das atividades de debates de teses, tornando-as mais fáceis de usar.

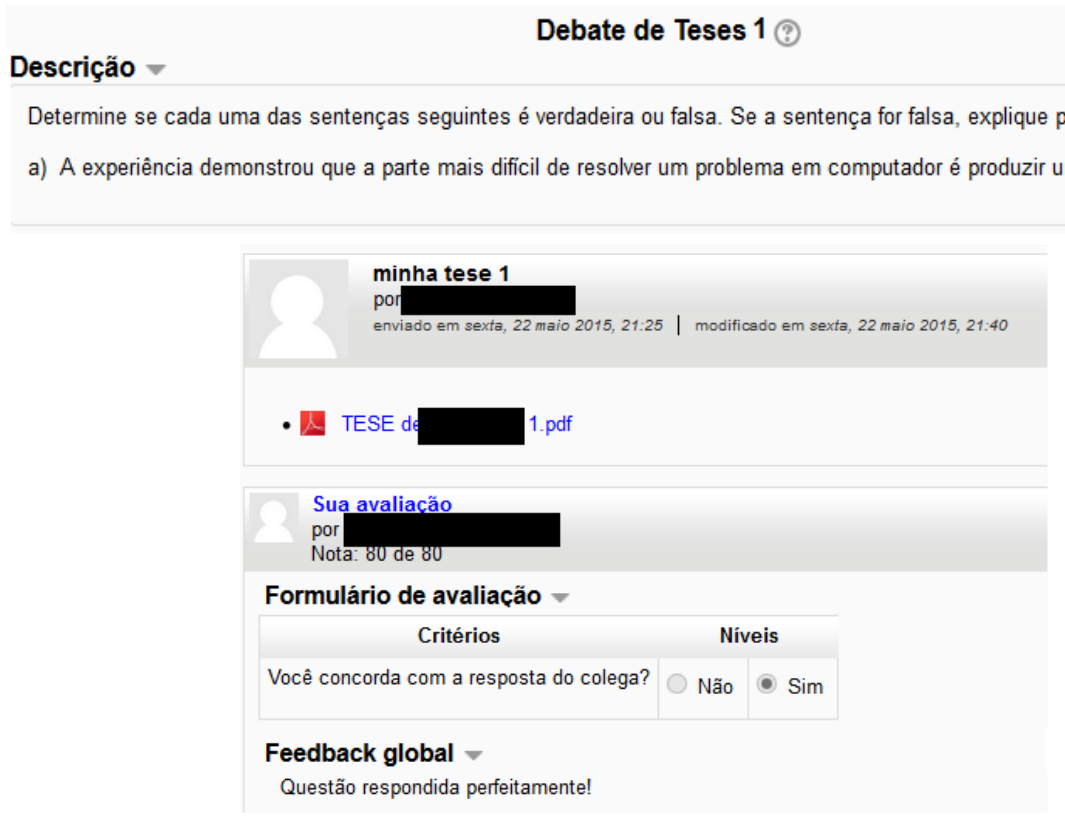

Figura 5. Exemplo da atividade debate de teses recomendada e sua avaliação por pares.

\section{Referências}

Araújo, R. D. et al. (2014). "Autoria automática de objetos de aprendizagem a partir de captura multimídia e associação a estilos de aprendizagem". Anais do XXV Simpósio Brasileiro de Informática na Educação, Dourados, MS.

Alonso, C. e Gallego, D. (2016) Questionário Honey-Alonso de Estilos de Aprendizagem, http://www.estilosdeaprendizaje.es/chaea/chaeagrafp2.htm.

Bremgartner, V.; Netto, J.M.; Menezes, C. S. (2015). "Explorando Arquiteturas Pedagógicas Recomendadas por meio de Agentes e Ontologia de Modelo do Aluno em Ambientes Virtuais de Aprendizagem". Anais do XXVI Simpósio Brasileiro de Informática na Educação, Maceió, AL.

Bull, S., Quigley, S. e Mabbott, A. (2006) "Computer-based Formative Assessment to Promote Reflection and Learner Autonomy". Engineering Education, vol. 1, no. 1, pp. 8-18.

Carvalho, M. J. S; Nevado, R. A.; Menezes, C. S. (2005). "Arquiteturas Pedagógicas para Educação a Distância: Concepções e Suporte Telemático". Anais - XVI Simpósio Brasileiro de Informática na Educação, 1, 362-372.

Castro, A. N. e Menezes, C. S. (2011) “Aprendizagem Colaborativa com Suporte Computacional”. In: Pimentel, M. e Fuks, H. Sistemas Colaborativos. Rio de Janeiro: Campus. ISBN: 978-85-352-4669-8.

CC/PP. (2016) Composite Capabilities/Preference Profiles, www.w3.org/Mobile/CCPP/

Felder, R. M. e Silverman, L. K. (1988). "Learning and teaching styles in engineering education". Engineering Education, 78, pp. 674-681.

FIPA. The Foundation for Intelligent Physical Agents. (2016). http://www.fipa.org/

Georgeff, M., Pell, B., Pollack, M., Tambe, M. e Wooldridge, M. (1999) "The Belief-Desire-Intention Model of Agency". In: Intelligent Agents V, LNAI 1555, Springer, pp. 1-10.

IEEE, LTSC. (2002). Draft Standard for Learning Technology - Learning Object Metadata. Technical report, IEEE Standards Department, New York.

JADE. (2016) Java Agent DEvelopment Framework, www.jade.tilab.com/

JADEX. (2016) JADEX Active Components, www.activecomponents.org/bin/view/About/Features

LIP. (2016) Learner Information Package Specification 1.0.1, www.imsglobal.org/profiles/lipinfo01.html\#5.1/ 
V Congresso Brasileiro de Informática na Educação (CBIE 2016)

Anais dos Workshops do V Congresso Brasileiro de Informática na Educação (CBIE 2016)

Marcon, K.; Machado, J. B.; Carvalho, M. J. S. (2012). "Arquiteturas pedagógicas e redes sociais: uma experiência no Facebook”. In: Anais do XVIII Congresso Brasileiro de Informática na Educação: Rio de Janeiro, RJ.

Moodle (2016) A Free, Open Source Course Management System for Online Learning, www.moodle.org/ Netto, J. F. M. (2006) "Uma Arquitetura para Ambientes Virtuais de Convivência: uma Proposta Baseada em Sistemas Multiagente". Tese de Doutorado, Universidade Federal do Espírito Santo, Vitória.

Panceri, S. S. e Menezes, C. S. (2014). "ALPES: Um sistema multiagente para análise de produções textuais no contexto de um Debate de Teses". Anais do XXV Simpósio Brasileiro de Informática na Educação (SBIE), Dourados, MS.

Protégé. (2016) The Ontology Editor, www.protege.stanford.edu/

Viccari, R. M. e Giraffa, L. M. M. (2003) "Fundamentos dos Sistemas Tutores Inteligentes". In: Barone, D. (Org). Sociedades artificiais: a nova fronteira da inteligência das máquinas. Porto Alegre: Bookman. ISBN: 85-363-0124-4. 